\title{
A New Power System Restoration Technique based on WAMS Partitioning
}

\author{
N. V. Phanendra Babu \\ Electrical Engineering Department, \\ National Institute of Technology \\ Warangal, Telangana, India
}

\author{
P. Suresh Babu \\ Electrical Engineering Department, \\ National Institute of Technology \\ Warangal, Telangana, India
}

\author{
D. V. S. S. Siva Sarma, \\ Electrical Engineering Department, \\ National Institute of Technology \\ Warangal, Telangana, India
}

\begin{abstract}
An important feature of a Wide-Area Measurement System (WAMS) is the ability to recover data during a communication failure. This paper presents a novel scheme of partitioning a PMU installed power network into a number of WAMS regions in order to make the power system restoration process simpler. This algorithm also proposes the optimal placement of Phasor Data Concentrators (PDCs) in each region to record the data from PMUs. This paper considers the restoration constraints like transformer equivalent bus, generation-load balance and the observability of region for the partitioning of power system. The proposed scheme is demonstrated with an IEEE-30 bus system. It is then applied on IEEE-39, IEEE-118 bus systems and on a Northern Regional Grid of the Indian Power Grid.
\end{abstract}

Keywords: wide-area monitoring system; WAMS; power system observability; power system restoration; phasor data concentrators; PDCs

\section{INTRODUCTION}

Blackouts are obviously the most dangerous outages that may happen in a power system, that call for immediate restoration [1]. This can be achieved by a restoration strategy called build-up strategy [2]. A delay in restoration causes damages to consumers and subjects the system to many economic and political costs. The build-up strategy recommended here follows a mutual interconnection of islands after restoring them separately. The build-up strategy and its importance are clearly explained in [2-5]. But sectionalizing a network into separate islands was not considered. A scheme was suggested in [6] to sectionalize a network into separate islands, but it failed to produce stable islands as it didn't considers the generation-load constraints during initial partitioning. Generally, during restoration, a circuit breaker between two buses should not be closed with a larger phase angle because it may damage the system by causing unwanted outages. Moreover, if the Phasor Measurement Unit (PMU) installed power system is partitioned, it may lose its observability because of the line openings between islands.

The present paper proposes a restoration based sectionalizing method for a PMU installed network. It is a model based algorithm that can handle even large-scale networks. Unlike [6], it considers power generation-load balance constraints during system initial partitioning. It also considers observability constraints at the final stage of the sectionalizing process. These two constraints have really increased the stability and observability of feasible islands. This would probably help power companies in protecting power systems from large catastrophes like blackouts just by isolating the islands that are being faulted. At the end it also suggests an optimal location strategy for Phasor Data Concentrators (PDCs) to provide phase angle data across the lines between any two islands. The proposed algorithm is demonstrated using the IEEE-30 bus system and then applied to the IEEE-39 and IEEE-118 bus systems and to a Northern Region of the Indian Power Grid.

\section{OPTIMAL PMU PLACEMENT}

A PMU gives synchronized measurements of electrical parameters like voltage, current and frequency of the bus on which it is installed. This section will optimize the number of PMUs required for observing the system completely. Actually, this optimization problem was introduced in [7]. Many heuristic methods like Genetic Algorithms [8], Particle Swarm Optimization [9], Hybrid Genetic Particle Swarm Optimization [10] and many others have been applied since. Meta-heuristic methods such as Binary Imperialistic Competition Algorithm (BICA) [11], Artificial Bee Colony (ABC) Algorithm [12] and Firefly Algorithm [13] have also been employed. An Integer Linear Programming (ILP) is developed in [14] for system complete observability. This was extended with and without conventional measurements in [15] and [16] respectively to include the zero-injection bus (ZIB) effect. Recently, researchers presented an integer quadratic based PMU placement policy [17]. An accurate solution for complete observability was presented in [18] using a participation factorbased method. In this paper it is assumed that the PMU placement has been conducted before partitioning the network. The Optimal PMU Placement problem is carried out using the Binary Cuckoo Search (BCS) technique. The PMU locations obtained are tabulated in Table I.

The problem is formulated as below.

Minimize

$$
\sum_{q \in N} x_{q}
$$

Subjected to 


$$
s_{p}(X) \geq 1, \forall p \in N(2)
$$

Where

$$
s_{p}=\sum_{q \in N} c_{p q} x_{q}, \forall p \in N
$$

Here, (1) is the objective function for the optimal PMU placement; $S_{p}$ represents observability function at bus $\mathrm{p}$. The parameter $c_{p q}$ from (3) gives the binary connectivity of all buses in the network. For the bus to be observable, it must be connected to at least on bus which is installed with PMU. The value of $S_{p}$ will be equal to or greater than 1 . It can be defined as,

$$
\mathcal{C}_{p q}=\left\{\begin{array}{l}
1, \text { if } p=q \\
1, \text { if } p \text { and q are connected } \\
0, \text { else }
\end{array}\right.
$$

TABLE I.

PMU LLOCATIONS

\begin{tabular}{|c|c|}
\hline $\begin{array}{c}\text { Test } \\
\text { system }\end{array}$ & \multicolumn{1}{|c|}{ Locations of PMUs } \\
\hline IEEE-30 & $2,3,6,9,10,12,15,19,25,27$ \\
\hline IEEE-39 & $2,6,9,10,12,14,17,20,22,23,26,29,33$ \\
\hline IEEE-118 & $\begin{array}{l}2,5,9,12,15,17,21,34,29,25,27,32,45,37,42,49,56,64, \\
\\
62,53,68,70,71,76,77,80,94,101,105,85,90,86,110\end{array}$ \\
\hline & $74,85,104,92,69,58,79,46,50,54,45,80,71,65,72,11,5$, \\
NRIP & $76,97,96,15,238,234,40,235,215,218,209,187,48,191,206$, \\
& $194,24,190,205,182,199,200,27,28,130,162,157,19,168$, \\
& $173,167,181,20,158,116,229,121,112,120,146,141,132$, \\
& $153,34,109,106,244,129,243,130,105,140,142$ \\
\hline
\end{tabular}

\section{CONSTRAINTS FOR WAMS POWER SYSTEM RESTORATION}

After sectionalizing the network, each island should be independently observable. For this, the process of network partition should be undergone three constraints like transformer equivalent bus, generation-load balance and observability of each island. The constraints will be explained as follows.

\section{A. Generation-Load balance}

This constraint ensures that there should be enough amount of active power to all the loads in each island. It can be represented as,

$$
\sum_{i=1}^{n_{r}} P_{G i}>\sum_{i=1}^{n_{r}} P_{L i}, i=1,2,3 \ldots . . \mathrm{r} .
$$

Where, $r$ represents the number of regions, $n_{r}$ represents the number of buses in each island, $P_{G i}$ gives maximum generation at $i^{\text {th }}$ bus and $P_{L i}$ gives the load at $i^{\text {th }}$ bus. This constraint must be satisfied in each-iteration of network partitioning.

\section{B. Joining the islands on either sides of transformer}

For the individual regions to run independently, it is required that the transformer and its buses should be allocated to the same region. This can be achieved by joining the islands present on either sides of transformers. It is a constraint that will be applied only after partitioning the complete system initially.

\section{Observability of islands}

This constraint will be checked by

$$
A_{p} \times x_{p} \geq\left[\begin{array}{l}
1 \\
1 \\
\cdot \\
\cdot \\
1
\end{array}\right]_{k \times k}
$$

Where, the matrix $A_{p}$ gives the system connectivity of the $p^{\text {th }}$ region. It can be calculated from (7). The vector $x_{p}$ of the $p^{\text {th }}$ region is the binary variable vector which can be calculated from (4), $k$ is the number of buses in the $p^{\text {th }}$ island. This is the last constraint that will be applied only after checking the second constraint. This constraint needs to be satisfied by each island, otherwise the island is said to be unobservable.

\section{PROPOSED NETWORK SECTIONALIZING}

Here, the partition method is illustrated by considering an IEEE-30 bus system as shown in Figure 1. After considering the system, the first step is to identify the number of islands. For this, let us consider the network connectivity matrix 'A' whose elements can be defined as,

$$
a_{i j}=\left\{\begin{array}{l}
1, \text { if } i=j \text { or buses } i \text { and } j \text { are connected } \\
0, \text { otherwise }
\end{array}\right.
$$

During the restoration, after the blackout, all the regions which are stable must be connected together to restore the system completely. Moreover, the number of islands decides the restoration time. So, while partitioning the system it should be assured that each region should have at least one generator and an allowable load connected to it. The number of regions can be selected as follows,

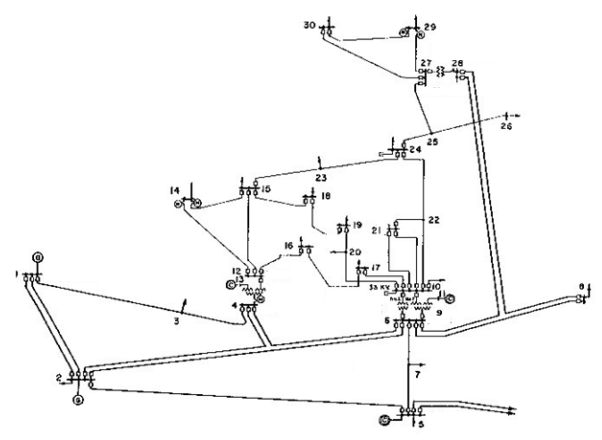

Fig. 1. IEEE-30 bus system

$$
r=\min \left\{\sum_{i=1}^{n} G_{i}, \sum_{i=1}^{n} L_{i}, \sum_{i=1}^{n} M\right\}
$$

Where,

$$
G_{i}=\left\{\begin{array}{l}
1, \text { if there is a generator at bus i connected } \\
\text { with an affordableload } \\
0, \text { otherwise }
\end{array}\right.
$$




$$
\begin{gathered}
L_{i}=\left\{\begin{array}{l}
1, \text { if there is a load bus at bus } i \\
0, \text { otherwise }
\end{array}\right. \\
M=\left\{\begin{array}{l}
1, \text { if bus i has } P M U \\
0, \text { otherwise }
\end{array}\right.
\end{gathered}
$$

After selecting the number of regions ( $r$ ), a matrix $R$ called regional matrix will be formulated. $\mathrm{R}$ is a $\mathrm{r} \times \mathrm{n}$ matrix in which each row corresponds to one region. Initially all elements in every-row of the matrix $\mathrm{R}$ set to 0 except for the buses with generator with affordable load and the corresponding load buses. Then, the next order regional matrix can be calculated using (12) given below.

$$
R_{k}=R_{k-1}^{\prime} \times A, k>1
$$

Where A is system connectivity matrix, $R_{k-1}^{\prime}$ is $R_{k-1}$ after corrections. This equation will be calculated iteratively until all the nodes (or buses) of the system were assigned. The corrections needed for higher order regional matrix will be defined as below:

1. The node once assigned, it should not be reassigned to any other region in the next iteration.

2. If any entry in the regional matrix is greater than unity, it should be corrected to 1 before the matrix is considered for next order regional matrix.

3. If a node appears in one region then it can be assigned to that particular region provided this correction should satisfy (5), otherwise the entry should be reset before it is being considered for next iteration.

4. If a node appears for more than one region, based on the nature of the bus it can be assigned to a region as follows:

Load bus:

- If a load bus appears in more than two regions, then it should be assigned to the one with the smaller power rating that could afford the load. The goal is to increase the number of regions (islands) and hence to reduce the restoration time.

- If there is no single region that could supply the load alone, the regions that are incident to load bus should be combined in order to supply the load.

Zero-Injection Bus (ZIB);

- If a ZIB appears in more than one regions then it should be assigned to the region with the smaller power rating. The goal is to increase the number of regions.

Generator bus:

- If a generator bus appears in more than one regions, then it is suggested to assign it to the region with the smaller power rating. The goal is to improve the stability of an island.

\section{FINAL PARTITION OF THE NETWORK}

For the islands to be observable, (6) needs to be verified. If it is not satisfied, it means some of the buses whose entries represent ' 0 ' need to be made observable by moving them into their adjacent islands provided the restoration constraints would not be disturbed. While making the unobservable buses observable, few situations that may arise will be handled as follows:

- Unobservable bus is a Load/Generator bus: yhe bus is then moved to an island via boundary line where it would be made observable provided this movement should not disturb the restoration constraints one and two. If this change causes violation of any constraint then the home island and the target island need to be joined.

- Unobservable bus is ZIB: If the unobservable bus is ZIB then it should be moved to a region where it would be observable.

The flowchart of the proposed methodology is shown in Figure 2.

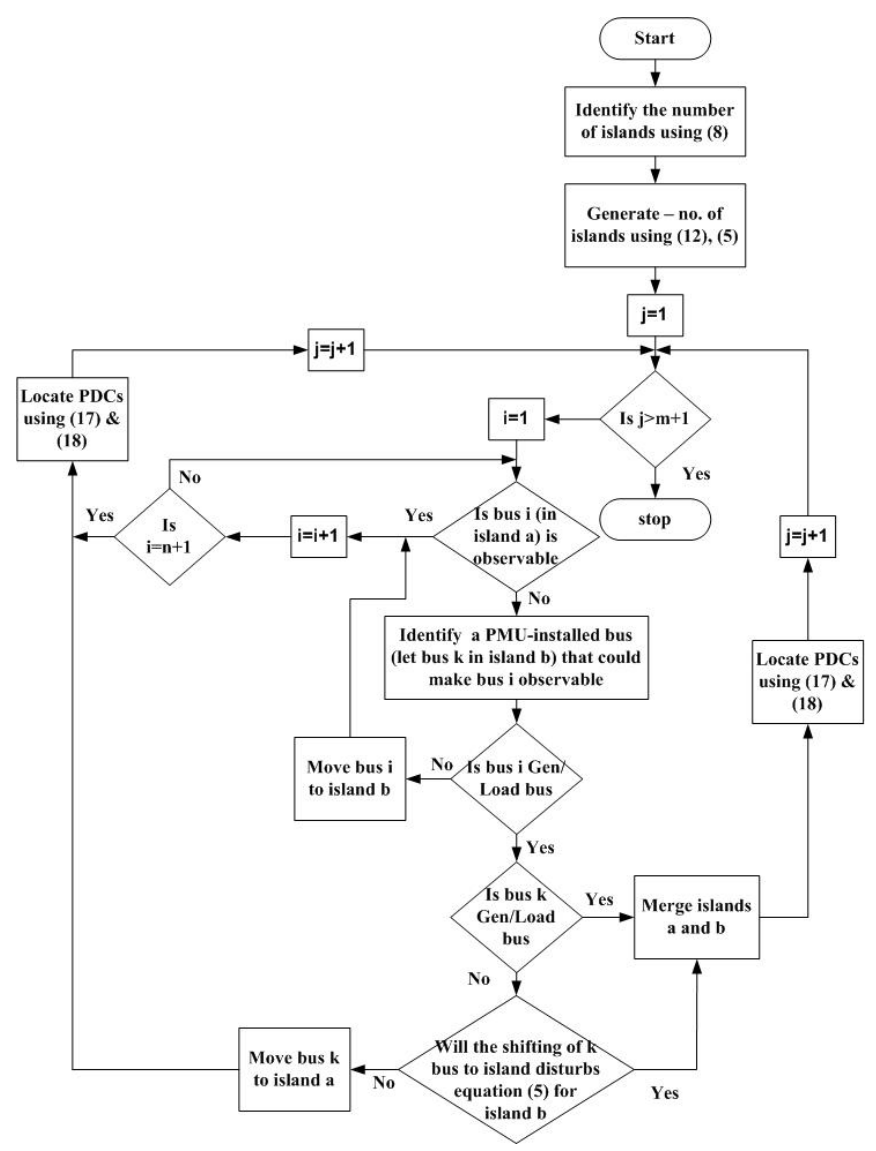

Fig. 2. Flow-chart of the proposed algorithm

\section{PHASOR DATA CONCENTRATOR PLACEMENT}

PDC archives the data from multiple PMUs installed over a wide area and aligns it for analytics /visualization. So, this must be placed in every island to provide certain functions like wide-area monitoring and protection. This section presents a new methodology to place PDCs in each island optimally with respect to the PMUs of that particular island. The problem of PDC placement can be defined as follows: 
Minimize

$$
S=\sum_{j=1}^{r} x_{p}
$$

Subjected to

$$
s_{p} \geq 1
$$

Where

$$
s_{p}=\sum_{i=1}^{n_{r}} c_{i j} x_{p}
$$

$$
c_{i j}=\left\{\begin{array}{l}
1, \text { if bus i has PMU, or j is PMU observed bus } \\
0, \text { itherwise }
\end{array}\right.
$$

Where, $x_{p}$ is a binary PDC placement variable, $s_{p}$ is a PMU placed constraint function at bus $\mathrm{p}, c_{i j}$ is a network path connectivity matrix. Once the network is partitioned, this method will be applied for all the islands.

\section{RESULTS AND DISCUSSIONS}

The partition method is illustrated by considering an IEEE30 bus system as shown in Figure 1. After identifying the number of islands as 6 , the initial regional matrix becomes $\mathrm{R}_{1}$ as shown in (17). And, the second order uncorrected regional matrix will be obtained as (18). On applying the corrections suggested in section 4 , the second order regional matrix of IEEE-30 bus system is obtained as (19). The final regional matrix is obtained as (20). From this equation it is quite clear that the regions $\mathrm{r} 3$ and $\mathrm{r} 5$ were combined so as to supply the load at bus 19 . So, the system is portioned into 5 stable regions as shown in Figure 3. The system presented in Figure 3 inherently exhibits generation-load balance as the first constraint is satisfied. Next, the regions that present on the either side of transformer need to be joined in order to satisfy the second restoration constraint. So, regions r2, r3 (or r5) will be joined.

$$
\begin{aligned}
& R_{1}=\left[\begin{array}{llllllllllllllllllllllllllllll}
1 & 0 & 1 & 0 & 0 & 0 & 0 & 0 & 0 & 0 & 0 & 0 & 0 & 0 & 0 & 0 & 0 & 0 & 0 & 0 & 0 & 0 & 0 & 0 & 0 & 0 & 0 & 0 & 0 & 0 \\
0 & 1 & 0 & 1 & 0 & 0 & 0 & 0 & 0 & 0 & 0 & 0 & 0 & 0 & 0 & 0 & 0 & 0 & 0 & 0 & 0 & 0 & 0 & 0 & 0 & 0 & 0 & 0 & 0 & 0 \\
0 & 0 & 0 & 0 & 0 & 0 & 0 & 0 & 0 & 0 & 0 & 1 & 1 & 0 & 0 & 0 & 0 & 0 & 0 & 0 & 0 & 0 & 0 & 0 & 0 & 0 & 0 & 0 & 0 & 0 \\
0 & 0 & 0 & 0 & 0 & 0 & 0 & 0 & 0 & 0 & 0 & 0 & 0 & 0 & 0 & 0 & 0 & 0 & 0 & 0 & 1 & 1 & 0 & 0 & 0 & 0 & 0 & 0 & 0 & 0 \\
0 & 0 & 0 & 0 & 0 & 0 & 0 & 0 & 0 & 0 & 0 & 0 & 0 & 0 & 1 & 0 & 0 & 0 & 0 & 0 & 0 & 0 & 1 & 0 & 0 & 0 & 0 & 0 & 0 & 0 \\
0 & 0 & 0 & 0 & 0 & 0 & 0 & 0 & 0 & 0 & 0 & 0 & 0 & 0 & 0 & 0 & 0 & 0 & 0 & 0 & 0 & 0 & 0 & 0 & 0 & 0 & 1 & 0 & 0 & 1
\end{array}\right] \\
& R_{2}^{\prime}=\left[\begin{array}{llllllllllllllllllllllllllllll}
1 & 1 & 1 & 0 & 0 & 0 & 0 & 0 & 0 & 0 & 0 & 0 & 0 & 0 & 0 & 0 & 0 & 0 & 0 & 0 & 0 & 0 & 0 & 0 & 0 & 0 & 0 & 0 & 0 & 0 \\
2 & 1 & 1 & 2 & 1 & 1 & 0 & 0 & 0 & 0 & 0 & 0 & 0 & 0 & 0 & 0 & 0 & 0 & 0 & 0 & 0 & 0 & 0 & 0 & 0 & 0 & 0 & 0 & 0 & 0 \\
0 & 0 & 0 & 1 & 0 & 0 & 0 & 0 & 0 & 0 & 0 & 2 & 2 & 1 & 1 & 1 & 0 & 0 & 0 & 0 & 0 & 0 & 0 & 0 & 0 & 0 & 0 & 0 & 0 & 0 \\
0 & 0 & 0 & 0 & 0 & 0 & 0 & 0 & 0 & 2 & 0 & 0 & 0 & 0 & 0 & 0 & 0 & 0 & 0 & 0 & 2 & 2 & 0 & 1 \rightarrow 0 & 0 & 0 & 0 & 0 & 0 \\
0 & 0 & 0 & 0 & 0 & 0 & 0 & 0 & 0 & 0 & 0 & 1 & 0 & 1 & 2 & 0 & 0 & 1 & 0 & 0 & 0 & 0 & 2 & 1 & 0 & 0 & 0 & 0 & 0 & 0 \\
0 & 0 & 0 & 0 & 0 & 0 & 0 & 0 & 0 & 0 & 0 & 0 & 0 & 0 & 0 & 0 & 0 & 0 & 0 & 0 & 0 & 0 & 0 & 0 & 1 & 0 & 2 & 1 & 2 & 2
\end{array}\right] \\
& R_{2}=\left[\begin{array}{llllllllllllllllllllllllllllll}
1 & 1 & 1 & 0 & 0 & 0 & 0 & 0 & 0 & 0 & 0 & 0 & 0 & 0 & 0 & 0 & 0 & 0 & 0 & 0 & 0 & 0 & 0 & 0 & 0 & 0 & 0 & 0 & 0 & 0 \\
0 & 1 & 0 & 1 & 1 & 1 & 0 & 0 & 0 & 0 & 0 & 0 & 0 & 0 & 0 & 0 & 0 & 0 & 0 & 0 & 0 & 0 & 0 & 0 & 0 & 0 & 0 & 0 & 0 & 0 \\
0 & 0 & 0 & 0 & 0 & 0 & 0 & 0 & 0 & 0 & 0 & 1 & 1 & 0 & 0 & 1 & 0 & 0 & 0 & 0 & 0 & 0 & 0 & 0 & 0 & 0 & 0 & 0 & 0 & 0 \\
0 & 0 & 0 & 0 & 0 & 0 & 0 & 0 & 0 & 1 & 0 & 0 & 0 & 0 & 0 & 0 & 0 & 0 & 0 & 0 & 1 & 1 & 0 & 1 \rightarrow 0 & 0 & 0 & 0 & 0 & 0 \\
0 & 0 & 0 & 0 & 0 & 0 & 0 & 0 & 0 & 0 & 0 & 0 & 0 & 1 & 1 & 0 & 0 & 1 & 0 & 0 & 0 & 0 & 1 & 0 & 0 & 0 & 0 & 0 & 0 & 0 \\
0 & 0 & 0 & 0 & 0 & 0 & 0 & 0 & 0 & 0 & 0 & 0 & 0 & 0 & 0 & 0 & 0 & 0 & 0 & 0 & 0 & 0 & 0 & 0 & 1 & 0 & 1 & 1 & 1 & 1
\end{array}\right] \\
& R_{4}=\left[\begin{array}{llllllllllllllllllllllllllllll}
1 & 0 & 1 & 0 & 0 & 0 & 0 & 0 & 0 & 0 & 0 & 0 & 0 & 0 & 0 & 0 & 0 & 0 & 0 & 0 & 0 & 0 & 0 & 0 & 0 & 0 & 0 & 0 & 0 & 0 \\
0 & 1 & 0 & 1 & 1 & 1 & 1 & 0 & 0 & 0 & 0 & 0 & 0 & 0 & 0 & 0 & 0 & 0 & 0 & 0 & 0 & 0 & 0 & 0 & 0 & 0 & 0 & 0 & 0 & 0 \\
0 & 0 & 0 & 0 & 0 & 0 & 0 & 0 & 0 & 0 & 0 & 1 & 1 & 1 & 1 & 1 & 1 & 1 & 1 & 1 & 0 & 0 & 1 & 0 & 0 & 0 & 0 & 0 & 0 & 0 \\
0 & 0 & 0 & 0 & 0 & 0 & 0 & 0 & 1 & 1 & 1 & 0 & 0 & 0 & 0 & 0 & 0 & 0 & 0 & 0 & 1 & 1 & 0 & 1 & 0 & 0 & 0 & 0 & 0 & 0 \\
0 & 0 & 0 & 0 & 0 & 0 & 0 & 0 & 0 & 0 & 0 & 1 & 1 & 1 & 1 & 1 & 1 & 1 & 1 & 1 & 0 & 0 & 1 & 0 & 0 & 0 & 0 & 0 & 0 & 0 \\
0 & 0 & 0 & 0 & 0 & 0 & 0 & 1 & 0 & 0 & 0 & 0 & 0 & 0 & 0 & 0 & 0 & 0 & 0 & 0 & 0 & 0 & 0 & 0 & 1 & 1 & 1 & 1 & 1 & 1
\end{array}\right]
\end{aligned}
$$


On applying the final portioning step for the system obtained above, the set of regions (r3, r6), (r2, r6) are combined via boundary lines because the target island (r6) individually cannot afford the loads at buses 24 and 8. Figure 4 represents the final network after all these modifications. And, it infers that the island $r 3$ covers larger. This is because of the fact that the generation in this region is accumulated where as the load is distributed.

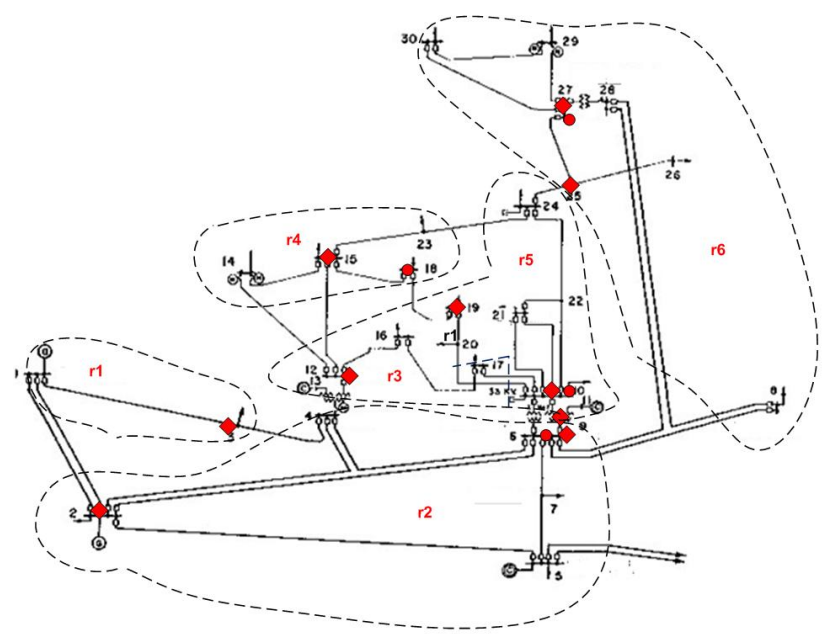

Fig. 3. Initial Partition of the IEEE-30 bus system

The locations of PDCs obtained on applying the PDC placement algorithm are shown in Table II. On applying the proposed scheme to a IEEE-39 system initially, it is clear that bus 18 is not observable and also it is found that it can be made observable by the PMU on bus 17 . Moreover, as the island $\mathrm{r} 2$ is able to afford the load at bus 18 , it has been moved to region r2. Figures 5 and 6 show the initial and final partitioning of IEEE-39 test system respectively. Table III gives the regions, PMU locations and PDCs locations after final partition.

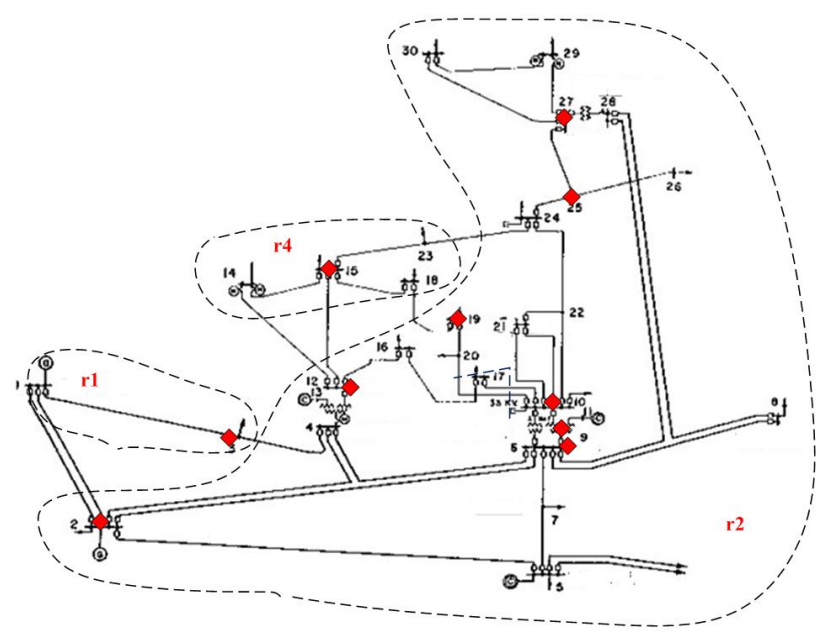

Fig. 4. Final Partition of the IEEE-30 bus system
TABLE II. REGIONAL INFORMATION AND PDCS LOCATIONS AFTER FINAL PARTITIONING IEEE-30 BUS SYSTEM

\begin{tabular}{|c|c|c|c|}
\hline Region & Buses & $\begin{array}{c}\text { PMU } \\
\text { locations }\end{array}$ & $\begin{array}{c}\text { PDC } \\
\text { locations }\end{array}$ \\
\hline r1 & 1,3 & 3 & 3 \\
\hline r4 & $14,15,18,23$ & 15 & 15 \\
\hline & $2,4,5,6,7,8,9,1,0$, & $2,6,9,10,12$, & $4,9,19,27$ \\
r2 & $11,12,13,16,17,19$, & $19,25,27$ & \\
& $20,21,22,24,25,26$, & & \\
& $27,28,29,30$ & & \\
\hline
\end{tabular}

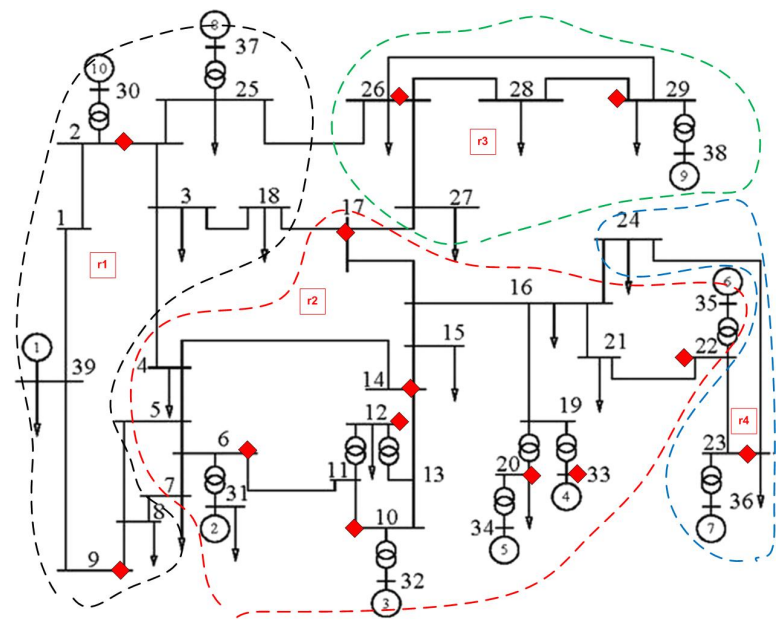

Fig. 5. Initial Partition of IEEE-39 bus system

TABLE III. REGIONAL INFORMATION AND PDCS LOCATIONS AFTER FINAL PARTITIONING IEEE-39 BUS SYSTEM

\begin{tabular}{|c|c|c|c|}
\hline Region & Buses & $\begin{array}{c}\text { PMU } \\
\text { locations }\end{array}$ & $\begin{array}{c}\text { PDC } \\
\text { locations }\end{array}$ \\
\hline $\mathrm{r} 1$ & $1,2,3,8,9,25,30,37,39$ & 2,9 & 2,9 \\
\hline $\mathrm{r} 2$ & $\begin{array}{c}4,5,6,7,10,11,12,13,14,15,16,17, \\
18,19,20,21,22,31,32,33,34,35\end{array}$ & $\begin{array}{c}6,10,12,14, \\
17,33,20\end{array}$ & $\begin{array}{c}11,14,17, \\
19,22\end{array}$ \\
\hline $\mathrm{r} 3$ & $26,27,28,29,38$ & 26,29 & 29 \\
\hline $\mathrm{r} 4$ & $23,24,36$ & 23 & 23 \\
\hline
\end{tabular}

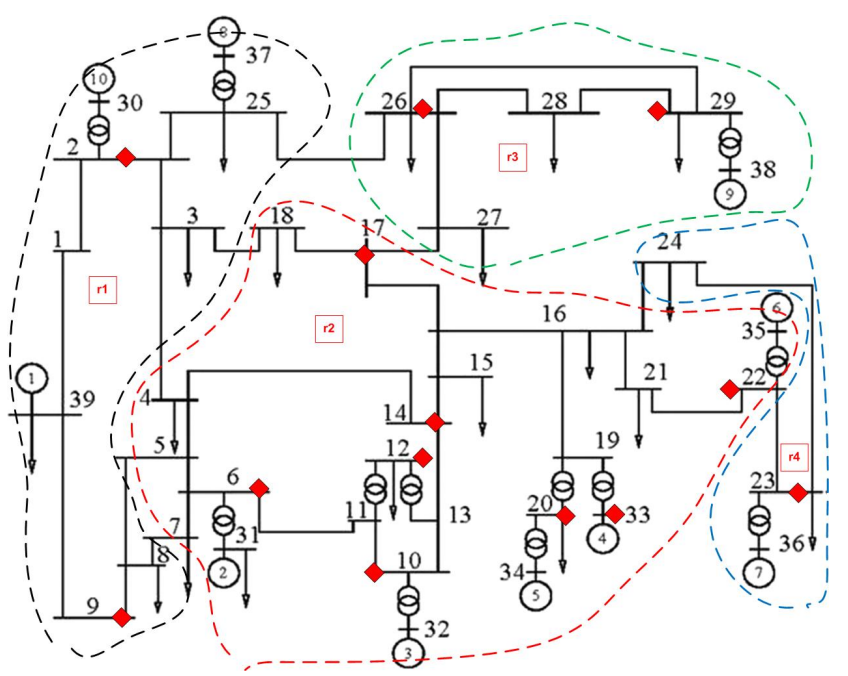

Fig. 6. Final Partition of IEEE-39 bus system 
Similarly for the IEEE-118 system, buses 48, 55, 59, 65, $78,95,106$ and 107 are found to be unobserved. Bus 48 is moved to the island containing bus 49 which could observe bus 48. Similarly, buses 78 and 95 have been moved to the regions containing buses 77 and 94 respectively. Even though the island with the PMU-installed bus 56 could observe buses 55, 59 and 65 , it cannot supply the required load at the respective buses. So, these two islands are combined. Similarly the island containing buses 106 and 107 is joined with an island that contains bus 105 which could make buses 105 and 107 observable. Figures 7 and 8 depict the initial and final partition of the IEEE-118 test system. Table IV contains the PMUs and PDCs location for different islands. An actual Northern Region of the Indian Power Grid (NRIP) has also been considered. Its final partition is shown in the Figure 9. The details of PMUs and PDCs are given in Table V. From the results, it is shown that this method has successfully produced more stable and observable islands which will really help countries which are yet to establish WAMS.

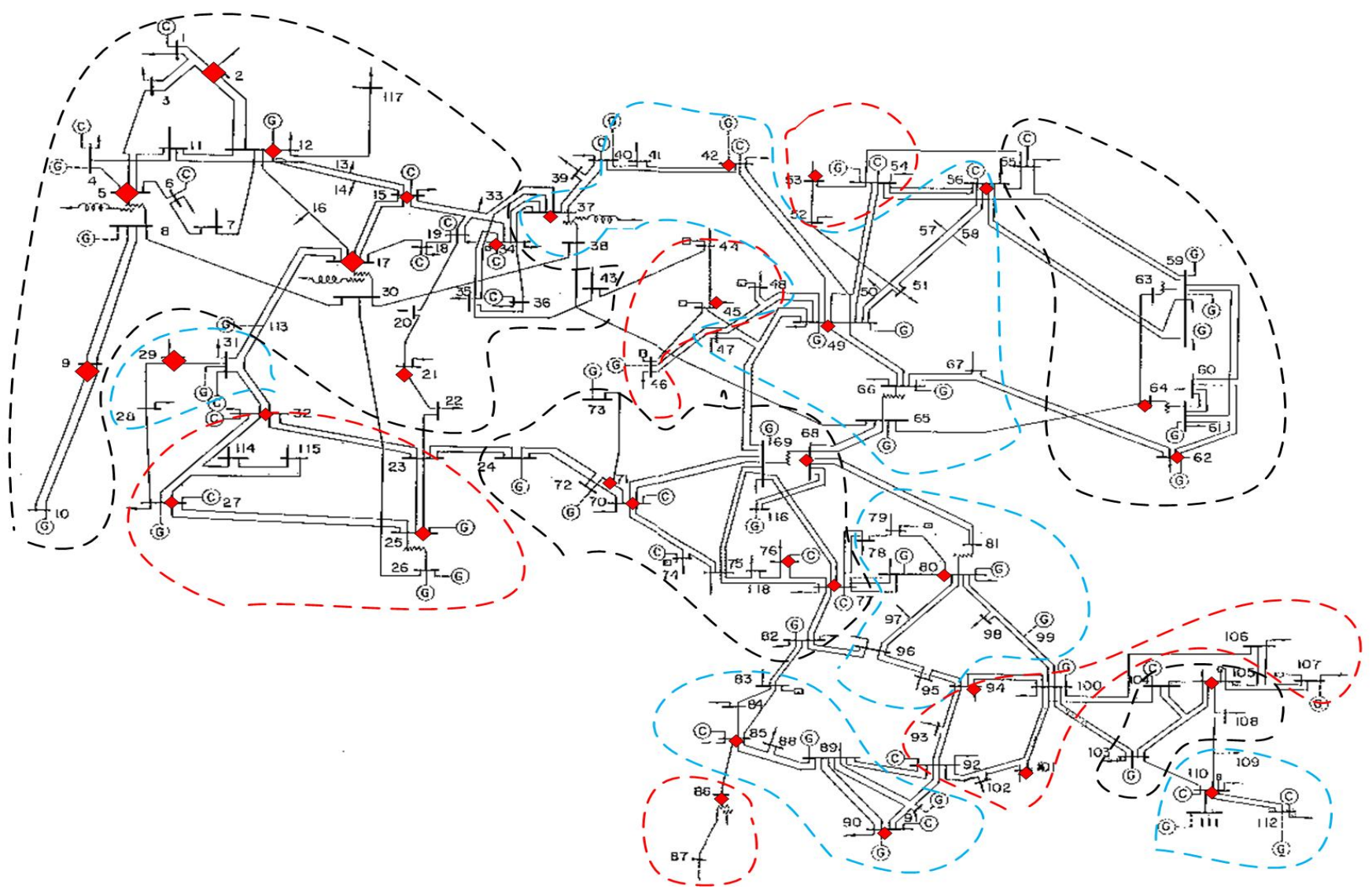

Fig. 7. Initial Partition of the IEEE-118 bus system

TABLE IV. REGIONAL INFORMATION AND PDCS LOCATIONS AFTER FINAL PARTITIONING IEEE-118 BUS SYSTEM

\begin{tabular}{|c|r|c|c|}
\hline Region & Buses & PMU locations & PDC locations \\
\hline r1 & $1,2,3,4,5,6,7,8,9,10,11,12,13,14,15,16,17,18,19,20,21,22,33,34,35,36,43,113,117$ & $2,5,9,12,15,17,21,34$ & $8,12,21,34$ \\
\hline r3 & $23,25,26,27,32,114,115$ & $25,27,32$ & 27 \\
\hline r6 & $24,47,68,69,70,71,72,73,74,75,76,77,78,116,118,82$ & $72,75,77,116$ & $25,69,71,115$ \\
\hline r2 & $28,29,31$ & 29 & 29 \\
\hline r5 & $37,38,39,40,41,42,47,48,49,50,51,55,56,57,58,59,60,61,62,63,64,65,66,67,68$ & $37,42,49,56,62,64$ & $40,66,56,64$ \\
\hline r4 & $44,45,46$ & 45 & 45 \\
\hline r7 & $52,53,54$ & 53 & 53 \\
\hline r8 & $79,80,81,96,97,98,99$ & 80 & 80 \\
\hline r9 & $83,84,85,88,89,90,91$ & $94,101,105$ & 100,105 \\
\hline 10 & 86,87 & 85,90 & 89 \\
\hline r1 & $109,110,111,112$ & 86 & 86 \\
\hline r12 & $92,93,94,95,100,10,102,103,104,105,107,108$ & 110 & 110 \\
\hline
\end{tabular}




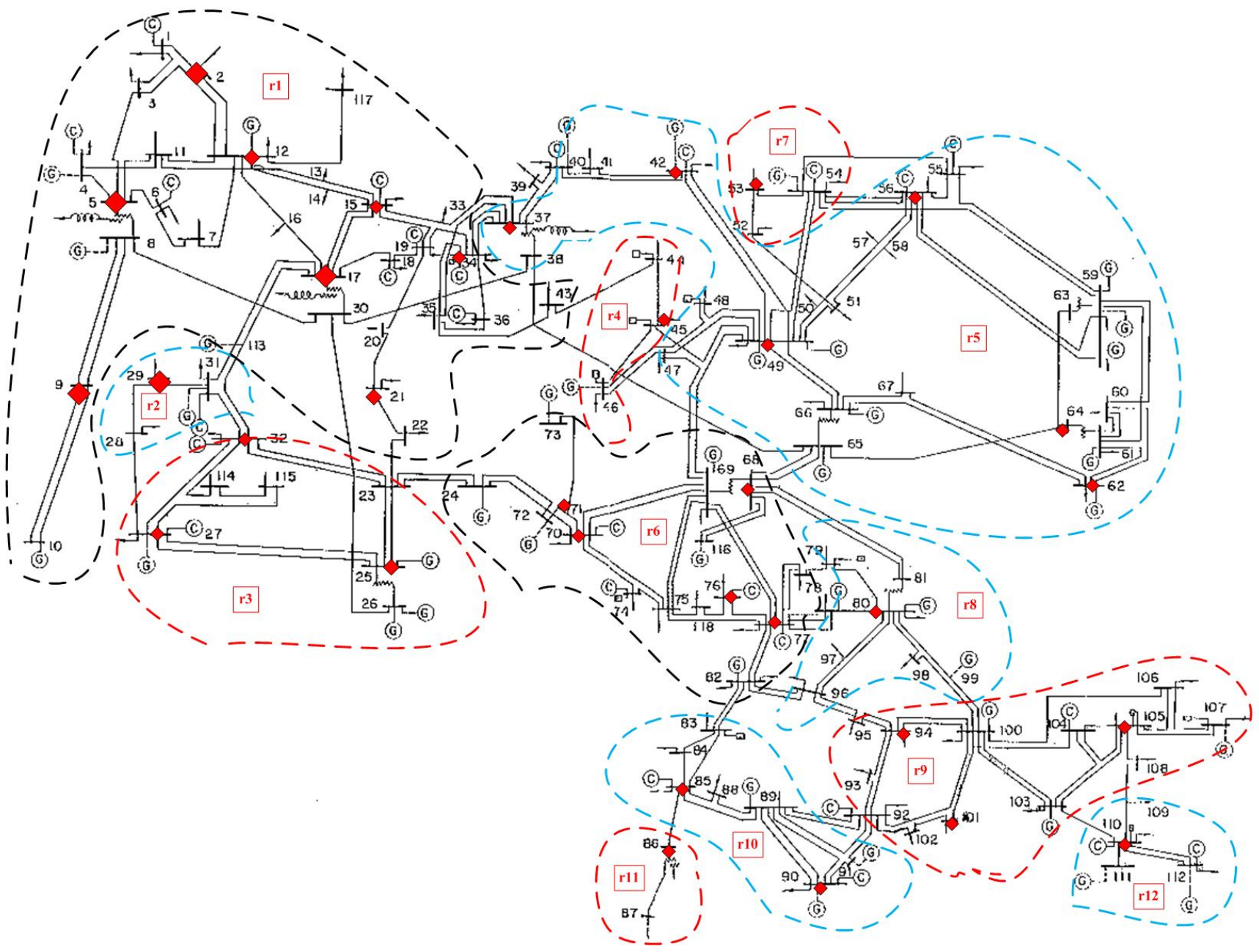

Fig. 8. Final Partition of the IEEE-118 bus system

TABLE V. REGIONAL INFORMATION AND PDCS LOCATIONS AFTER FINAL PARTITIONING NRIP

\begin{tabular}{|c|c|c|c|}
\hline Region & Buses & PMU locations & PDC locations \\
\hline $\mathrm{r} 1$ & $\begin{array}{c}84,246,94,69,131,10,8,88,74,103,85,102,83,104,95,78,92,12,82,86,91,75,70 \\
89,80,44,57,25,46,64,55,63,39,7,67,56,58,51,54,52,49,62,4,43,71,3,72,73,179 \\
235,60,9,11,13,5,90,96,87,100,110,66,98,17,93,15,99,101,76\end{array}$ & $\begin{array}{l}74,85,104,92,80,71,45,46,5 \\
0,54,58,69,79,65,5,11,72,7 \\
6,97,115,96\end{array}$ & $\begin{array}{l}5,11,49,56,58,70 \\
76,78,79,90,95 \\
103\end{array}$ \\
\hline r2 & $\begin{array}{c}40,41,32,33,218,215,211,59,214,212,217,216,213,196,77,31,30,209,2,189,187, \\
204,47,219,197,185,184,208,210,186,207,206,48,210,220,124,190,29,188,221, \\
191,192,195,220,183,194,24,226,222,223,225,205,227,133,228,26,203,224,39, \\
1,152,200,231,27,202,28,198,159,30,231,200,201,28,199\end{array}$ & $\begin{array}{l}40,215,218,187,48,206,219 \\
191,194,24,190,205,182,1 \\
99,200,27,28\end{array}$ & $\begin{array}{l}29,47,48,152,191 \\
194,196,199,202 \\
211,218\end{array}$ \\
\hline r3 & $\begin{array}{c}23,180,22,157,162,176,177,161,175,21,19,166,178,169,173,167,163,167,181 \\
172,230,164,160,168,174,165,158,171,170,37,20,156\end{array}$ & $\begin{array}{c}180,187,162,19,168,20 \\
173,167,158,181\end{array}$ & $\begin{array}{l}21,157,166,170 \\
181\end{array}$ \\
\hline r4 & $\begin{array}{c}240,235,38,233,24,239,238,234,237,230,36,118,232,130,105,242,42,129,126 \\
127,114,105,107,243,108,120,113,123,244,245,131,135,109,112,122,16,125 \\
124,106,34,150,153,132,139,143,152,240,144,121,229,136,138,141,150,153 \\
149,147,140,18,142,145,142,154,61,119,117,35,17,134,146,148,53,155,111 \\
116,151,137,128\end{array}$ & $\begin{array}{l}235,234,238,130,105,129,1 \\
20,112,121,229,106,244,24 \\
3,109,34,141,146,153,132 \\
\quad 140,142,116\end{array}$ & $\begin{array}{c}35,106,108,118 \\
122,126,128,137 \\
140,147,235,240\end{array}$ \\
\hline
\end{tabular}

\section{CONCLUSION}

This paper assures a faster restoration process by partitioning the network into many regions which are easier to be restored during a build-up strategy. Initially this partition starts with sectionalizing the network subjecting to the generation-load balance constraint, and next these sections are reorganized to ensure the remaining two restoration 
constraints. This approach provides synchronized phase angle data between any two adjacent islands thus ensuring the complete observability of all regions. This partition algorithm was applied on three different systems and sectionalized the
IEEE-39 system into four regions, the IEEE-118 system into 12 islands and a Northern Region of the Indian Power Grid into 4 stable islands.

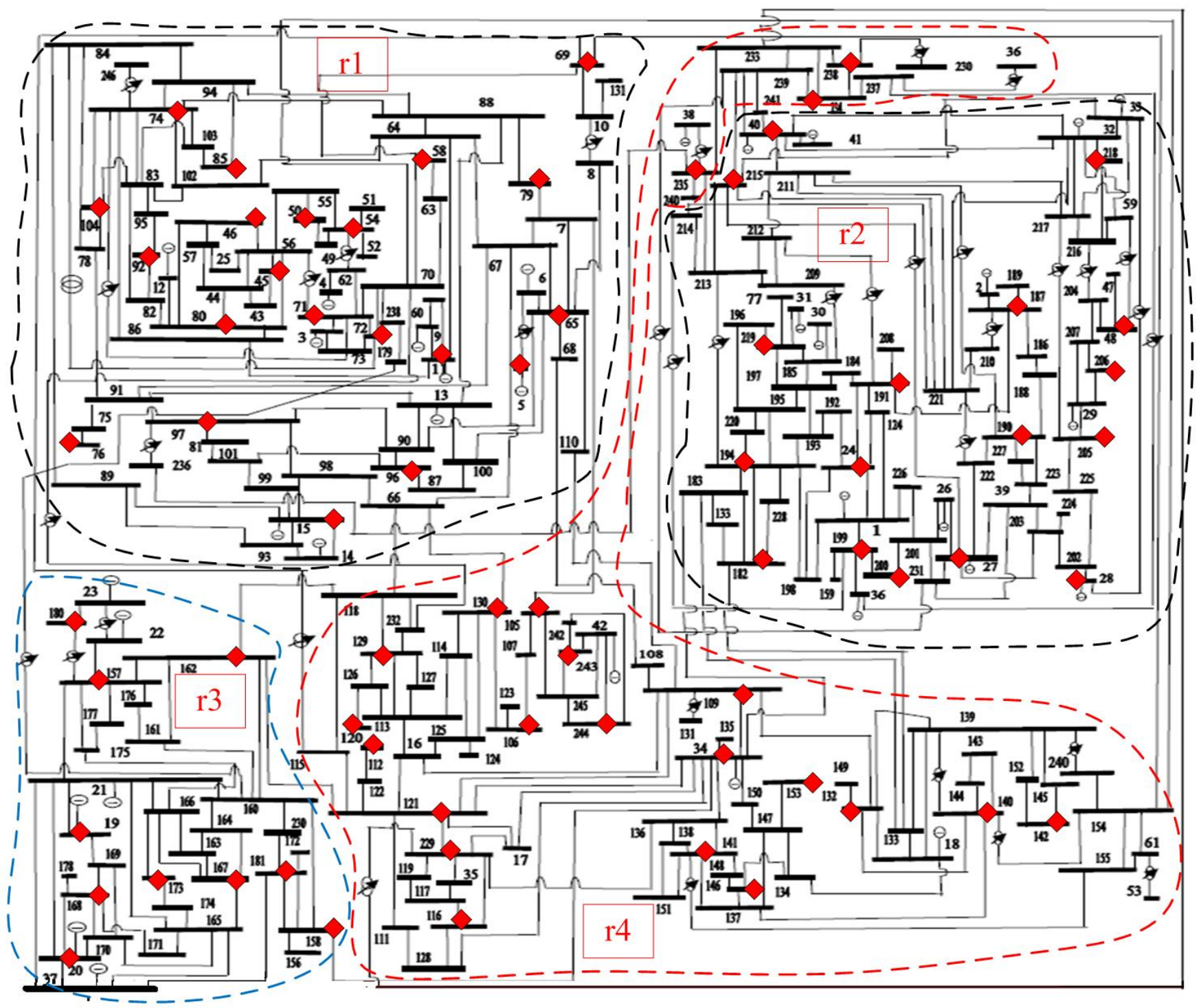

Fig. 9. Final Partitions of the NRIP system

\section{REFERENCES}

[1] S. Tamronglak, S. E Horowitz, A. G. Phadke, J. S. Thorp, "Anatomy of power system blackouts: preventive relaying strategies", IEEE Transactions on Power Delivery, Vol. 11, No. 2, pp. 708-715, 1996

[2] M. Adibi, L. H. Fink, "Power system restoration planning", IEEE Trans. Power Syst., Vol. 9, No. 1, pp. 22-28, 1994

[3] F. Wu, A. Monticelli, "Analytical tools for power system restorationConceptual design”, IEEE Trans. Power Syst., Vol. 3, No. 1, pp. 10-16, 1988
[4] R. J. Kafka, D. R. Penders, S. H. Bouchey, M. M. Adibi, "System restoration plan development for a metropolitan electric system", IEEE Trans. Power App. Syst., Vol. PAS-100, pp. 3703-3713, 1981

[5] J. A. Huang, F. D. Galiana, G. T.Vuong, "Power system restoration incorporating interactive graphics and optimization", Proc. 14th Int. Conf. Power Ind. Appl., Baltimore, MD, 1991

[6] S. A. N. Sarmadi, A. S. Dobakhshari, S. Azizi, A. M. Ranjbar, "A Sectionalizing Method in Power System Restoration Based on WAMS", IEEE Transactions on Smart Grids,Vol. 2, No. 1, 2011

[7] T. L. Baldwin, L. Mili, M. B. Boisen,R. Adapa, "Power system observability with minimal phasor measurement placement", IEEE Trans. Power Syst., Vol. 8, No. 2, pp. 707-715, 1993 
[8] F. J. Marin, F. Garcia-Lagos, G. Joya,F. Sandoval, "Genetic algorithms for optimal placement of phasor measurement units in electric networks", Electron. Lett., Vol. 39, No. 19, pp. 1403-1405, 2003

[9] M. Hajian, A. M. Ranjbar, T. Amraee, A. R. Shirani, "Optimal placement of phasor measurement units: particle swarm optimization approach", Proc. Int. Conf. Intelligent Systems Application Power Systems, pp. 1-6, November 2007

[10] N. V. Phanendra Babu, P. Suresh Babu, D. V. S. S. Siva Sarma, “A Reliable Wide-Area Measurement System Using Hybrid Genetic Particle Swarm Optimization (HGPSO)", International Review of Electrical Engineering, Vol. 10, No. 6, pp. 747-763, 2015

[11] A. Mahari, H. Seyedi, "Optimal PMU placement for power system observability using BICA, considering measurement redundancy", Electric Power Systems Research, Vol. 103,pp. 78- 85, 2013

[12] A. Kulanthaisamy, R. Vairamani, N. K. Karunamurthi, C. Koodalsamy, "A Multi-objective PMU Placement Method Considering Observability and Measurement Redundancy using ABC Algorithm”, Advances in Electrical and Computer Engineering, Vol. 14, No. 2, pp. 117 - 128, 2014

[13] K. Arul Jeyaraj, V. Rajasekaran, S. K. Nandha Kumar, K. Chandrasekaran, "A Multi-objective Placement of Phasor Measurement Units Considering Observability and Measurement Redundancy Using Firefly Algorithm", J. Electr. Eng. Technol., Vol. 10, No. 2, 474-486, 2015.
[14] B. Xu, A. Abur, "Observability analysis and measurement placement for system with PMUs", IEEE Power Systems Conf. Exposition, , Vol. 2, pp. 943-946, October 2004

[15] B. Gou, "Optimal placement of PMUs by integer linear programming", IEEE Trans. Power Syst., Vol. 23, No. 3, pp. 1525-1526, 2008

[16] B. Gou, "Generalized integer linear programming formulation for optimal PMU placement”, IEEE Trans. Power Syst., Vol. 23, No. 3, pp. 1099-1104, 2008

[17] S. Chakrabarti, E. Kyriakides, D. G. Eliades, "Placement of synchronized measurements for power system observability," IEEE Trans. Power Del., Vol. 24, No. 1, pp. 12-19, 2009

[18] E. Caro, R. Singh, B. C. Pal, A. J. Conejo, R. A. Jabr, "Participation factor approach for phasor measurement unit placement in power system state estimation", IET Gener. Transm. Distrib., Vol. 6, No. 9, pp. 922929,2012 